\title{
Editorial: About the Foodborne Pathogen Campylobacter
}

\author{
Odile Tresse ${ }^{1 *}$, Avelino Alvarez-Ordóñez ${ }^{2}$ and lan F. Connerton ${ }^{3}$ \\ ${ }^{1}$ SECALIM, INRA, Oniris, Université Bretagne Loire, Nantes, France, ${ }^{2}$ Department of Food Hygiene and Technology and \\ Institute of Food Science and Technology, University of León, León, Spain, ${ }^{3}$ Division of Food Sciences, School of \\ Biosciences, University of Nottingham, Nottingham, United Kingdom
}

Keywords: foodborne pathogen, epidemiology, host interaction, control strategies, metabolism and regulation, methodology, chicken gut microbiota

\section{Editorial on the Research Topic}

\author{
About the Foodborne Pathogen Campylobacter
}

\section{INTRODUCTION}

The name "Campylobacter" comes from ancient Greek meaning "curved rod" which describes the shape of this microorganism. Campylobacter was firstly isolated as a Vibrio species from epizootic ovine abortion in 1906 by McFadyean and Stockman (1913), and renamed in 1973 as the neotype strain Campylobacter after showing significant biological differences with Vibrio species (Véron

OPEN ACCESS

Edited by:

David Rodriguez-Lazaro, University of Burgos, Spain

Reviewed by: Alessandra De Cesare, Università di Bologna, Italy

Paula Teixeira, Universidade Católica Portuguesa,

Portugal

${ }^{*}$ Correspondence: Odile Tresse odile.tresse@oniris-nantes.fr

Specialty section: This article was submitted to

Food Microbiology,

a section of the journal

Frontiers in Microbiology

Received: 28 July 2017 Accepted: 19 September 2017 Published: 10 October 2017

Citation:

Tresse O, Alvarez-Ordóñez A and Connerton IF (2017) Editorial: About

the Foodborne Pathogen Campylobacter

Front. Microbiol. 8:1908.

doi: 10.3389/fmicb.2017.01908 and Chatelain, 1973). Rather than a curved rod, the shape looks more like to a spiral and can develop in to filamentous or coccoid forms under stressful conditions (Tangwatcharin et al., 2006; Ghaffar et al., 2015; Rodrigues et al., 2016). Nowadays, Campylobacter spp. are classified among the $\varepsilon$-proteobacteria in the family of Campylobacteriaceae (Vandamme et al., 1991). Campylobacter has emerged as the leading cause of bacterial foodborne infections in developed countries, having surpassed Salmonella several years ago, and represents a significant economic burden (EFSA and ECDC, 2016). Although new species of Campylobacter have been recently discovered, human cases of campylobacterosis are dominated by two main species, Campylobacter jejuni and, to a lesser extent, Campylobacter coli.

Quantitative epidemiology reports reveal high rates of contamination for broiler chickens and carcasses by Campylobacter (Hue et al., 2010; Lawes et al., 2012; Powell et al., 2012). The presence of Campylobacter was also detected in other farm animals or foodstuffs due to cross contamination (EFSA and ECDC, 2016). Campylobacter in poultry remains a problem with no effective control measures available that can be recommended for microbial food/farm safety guidelines to mitigate the risk of flock colonization. Campylobacter also remains a puzzle as to how an obligate microaerobic bacterium can survive from farm to retail outlets. The underlying molecular mechanisms of persistence, survival and pathogenesis appear to represent a combination peculiar to this pathogen, which are not shared with other foodborne bacterial pathogens such as Listeria monocytogenes, Salmonella enterica, Escherichia coli, and Staphylococcus aureus.

This topic includes 18 published articles describing original studies of C. jejuni and C. coli that deal with (1) epidemiology and animal carriage, (2) host interaction, (3) control strategies, (4) metabolism and regulation specificities of these two pathogen species, (5) methodology to improve cultural technique and (6) chicken gut microbiota challenged with Campylobacter.

\section{Epidemiology and Animal Carriage}

Organic animal production schemes differ in many ways (antibiotic use, herd structure, feeding regimes, access to outdoor areas, space allowance) from conventional rearing systems, and therefore can have an impact in the occurrence, transmission and pathogenicity of foodborne 
pathogens, including Campylobacter spp. In this research topic, is however shown that organic pig production schemes have a minor impact on the epidemiology of C. coli. Kempf et al. monitored the prevalence and antimicrobial resistance of C. coli isolated from conventional and organic pigs in farms from France and Sweden and observed no significant difference in prevalence between pigs in organic and conventional productions. They however observed in France a higher occurrence of antimicrobial resistant C. coli isolates (particularly against the antibiotics tetracycline and erythromycin) from conventional rearing systems. Denis et al. characterized C. coli isolates obtained from 19 organic pig farms and 24 conventional pig farms through pulsed field gel electrophoresis, multilocus sequence typing, detection of nine virulence-associated genes and evaluation of the adhesion and invasion capacity on Caco- 2 cells. They concluded that pig farm management strategies did not influence the diversity and virulence of C. coli.

\section{Host Interactions}

Ayllón et al. used a proteomic approach to examine relative differences in protein expression levels between C. jejuni interacting with human (INT-407) and porcine (IPEC-1) cell lines. The study revealed 366 differentially expressed proteins after $3 \mathrm{~h}$ infection and 485 after $24 \mathrm{~h}$. The identities of the protein enabled analysis of the response pathways that indicate differences in the timing of inflammatory responses between the cell lines and comparative down regulation of the signaling pathways that control cell migration, endocytosis and cell cycle progression in the porcine cell line. The authors attribute the differences in the cellular pathway responses to C. jejuni exposure as indicative of the processes that establish either infective or commensal behavior respectively in human or porcine hosts.

Upadhyay et al. examined the potential of three phytochemicals generally recognized as safe (GRAS) and applied at sub-inhibitory concentrations to bacterial growth, to prevent or reduce the severity of human infection. Phytochemical treatments of C. jejuni resulted in reduced motility and a reduction in the expression of cytolethal distending toxin that could result in favorable effects on human infection. Using human intestinal epithelial (Caco-2) cell-based assays the authors demonstrated the abilities of trans-cinnamaldehyde $(0.01 \%)$, carvacrol $(0.002 \%)$, and eugenol $(0.01 \%)$ to reduce the processes of attachment, invasion and translocation of $C$. jejuni.

\section{Control Strategies}

Effective control of Campylobacter on commercial broiler chicken farms is proving more than challenging. Microbiological risk assessments suggest that if reductions in the intestinal loads carried by chickens could be translated on to poultry meat then these measures could make a significant impact on the cases of human campylobacterosis (Boysen et al., 2013). Two studies within the topic report the results of feed supplement additions to broiler chicken diets directed to reduce Campylobacter colonization levels. Guyard-Nicodème et al. reported the effects of five treatments applied on a French free-range broiler farm that became naturally positive for $C$. jejuni when the birds went on to range after 35 days. A combination treatment of a cation exchange clay-based product and an organic acid mixture containing formic acid, sodium formate, lactic acid and propionic acid resulted in a modest but significant reduction of $\log _{10} 0.82$ CFU per $\mathrm{g}$ of cecal contents at day 78 when the birds were scheduled for processing. This treatment was also found to be associated with a significant reduction of $\log _{10} 0.68 \mathrm{CFU}$ per $\mathrm{g}$ on the neck skins of the carcasses of the chickens. Wagle et al. examined the impact of $\beta$-resorcylic acid as in-feed supplement and the impact of the phytophenolic on the in vitro infection of Caco-2 intestinal epithelial cells. Broiler chickens were challenged with $C$. jejuni at day 7 and fed $\beta$-resorcylic acid supplemented feed $(0.25$ to $1 \%)$ until day 14 , which resulted in significant reductions up to $\log _{10} 2.5 \mathrm{CFU}$ per g of cecal contents. Subinhibitory concentration also reduced C. jejuni motility and their ability to attach and invade Caco-2 cells.

Bacteriophage therapy is a sustainable biological control that has the potential to reduce Campylobacter colonization of broiler chickens (Connerton et al., 2011). Lis and Connerton investigated factors that impact on virulent bacteriophage infection of $C$. jejuni. As discussed above motility is a critical trait for successful intestinal colonization and infection by C. jejuni. These studies confirmed that motility is also critical for one class of bacteriophage and required for efficient infection by a second. For C. jejuni any loss in motility to escape phage infection would result in an inability to colonize animal hosts. As a response to this Achilles heel, campylobacters utilize a second minor flagellin protein, FlaB, to enable regrowth post phage infection. These populations arise still largely at the expense of motility but have the potential to revert to full motility and survive once separated from the bacteriophage. Although, once motility has been recovered, they will become susceptible once more to bacteriophage infection.

\section{Metabolism and Regulation Glucose Metabolism}

C. jejuni is an asaccharolytic micro-organism characterized by the absence of a functional Embden-Meyerhof-Parnas glycolysis pathway due to the absence of glucokinase (Glk) and phosphofructokinase (Pfk). Gluconeogenesis fueled by amino acids is the main pathway for $C$. jejuni to synthesize anabolic hexose phosphate. The Entner-Doudoroff (ED) pathway is an alternative in bacteria to synthesize pyruvate from extracellular glucose via phosphogluconate in order to bypass the absence of Pfk. In this research topic, the key genes of the ED pathway have been found in the genomes of rare isolates of both C. jejuni and C. coli (Vegge et al.). A complete gene set encoding a functional ED pathway in these C. jejuni and C. coli isolates are located on a transferable genomic island, similar to that for the genes involved in the utilization of L-fucose in human gut by few C. jejuni (Stahl et al., 2011). Interestingly, the presence of a functional ED pathway gives advantages to these bacterial isolates for survival and biofilm formation (Vegge et al.).

\section{Oxidative Stress Response}

Campylobacter species are obligate microaerobic microorganisms characterized by the inability to multiply in ambient levels of oxygen (Macé et al., 2015). However, some 
strains are able to better counteract aerobic conditions for their survival. Recently, the atypical C. jejuni $\mathrm{Bf}$ was described to be able to grow under aerobic conditions in contrast to other C. jejuni (Rodrigues et al., 2015, 2016). As for C. jejuni Bf, C. coli OR12 is also able to develop an habituation to aerobic conditions. In this research topic, the hyperaerotolerance of C. coli was found to be associated with an increased resistance to peroxide stress (O'Kane and Connerton). On the other hand, C. jejuni is not able to grow anaerobically, even though it can produce functional alternative electron acceptors to $\mathrm{O}_{2}$, such as fumarate, nitrate, nitrite, trimethylamine-N-oxide or dimethylsulfoxide (Weingarten et al., 2009). This was attributed to the absence of an alternative to the $\mathrm{O}_{2}$-dependent Class I type of ribonucleotide reductase, an enzyme essential for DNA synthesis (Sellars et al., 2002). In this research topic, the regulation and function of various $\mathrm{C} 4$-dicarboxylate components as alternative electron acceptors or transporters in C. jejuni were explored. It was suggested that the DctA transporter participates in the uptake of succinate at high oxygen levels while $d c u A$ and $d c u B$ genes, controlled by RacRS system, are up-regulated in oxygen-limited conditions (Wösten et al.). The main enzymes constituting the sub-system of oxygen detoxification in C. jejuni has been described. Nonetheless, the regulation of these enzymes remain elusive as the main regulators previously described to play this role in other Gram-negative bacteria (SoxRS and OxyR regulons) are absent in $C$. jejuni genome. These enzymes could be potentially controlled by the iron homeostasis and transcriptional regulating mechanisms including the essential pleiotropic regulator CosR and the inessential RrpA and RrpB regulators. In this research topic, Gundogdu et al. have brought new insights concerning the roles of RrpA and $\operatorname{RrpB}$ in the response to oxidative and aerobic stress conditions. The gene $\operatorname{rrp} A$ is present in over $99 \%$ of $C$. jejuni strains while $\operatorname{rrpB}$ seems to be restricted to livestock clonal complexes. This latter gene is located to a transferable hypervariable region in association with the type I R-M ( $h s d)$ system. Consequently, the presence of RrpB suggests a specific adaptation of $C$. jejuni to host.

\section{Phase Transition}

Phase transition in bacteria is important for survival and adaptation to harmful conditions. As mentioned earlier, C. jejuni is able to modulate its shape in response to environmental conditions. The life cycle of Campylobacter is an alternation of states from dormancy to multiplication. Bacterial growth is also punctuated by different phases. The lag phase represents the time for the bacteria to adapt to new conditions, the log phase is characterized by the cell doubling and the stationary phase is a way of survival in growth limiting conditions. The timing of the latter phase is conditioned by the adaptation capability of the cells. It is usually driven by growth factors in response to general stress. In contrast to most of Gram-negative bacteria, the main transcriptional factor, RpoS, which controls the switch between the log and the stationary phases, is absent in Campylobacter. Reported in this research topic is the identification of proteins showing significantly different abundances between the two growth phases (Turonova et al.). These proteins belong to biological pathways including metabolism, general and specific oxidative stress response, translation and motility. In addition, the regulator CosR was identified among these differently abundant proteins. The dynamics of the transcript levels of CosR throughout the growth of $C$. jejuni reveal transient differences between the log and stationary phases, suggesting the transcriptional regulator is under negative control. As aforementioned, CosR was originally ascribed a role in the control of enzymes involved in oxygen detoxification. Further analyses indicated that $\operatorname{Cos} \mathrm{R}$ is able to bind to its own promoter region indicating its potential for auto-regulation. The DNA binding consensus sequence of CosR was refined by bioinformatic analysis of the promotor region of CosR and other genes previously described to be able to bind this protein. Although the complete regulatory framework associated with CosR remains to be discovered, these data suggest a major contribution of CosR during the switch between exponential and stationary phases in C. jejuni.

\section{Characterization of New Genes}

Whole genome sequencing provides comprehensive set of features by which Campylobacter demonstrates genetic variation and plasticity. Many well described molecular mechanisms in bacteria are not transferable to Campylobacter due to the absence of homologous genes. Consequently, the mutational analysis of specific genes with putative functions continues to turn up new aspects of the biology of Campylobacter. For instance, the putative transcriptional regulator Cj0440c, belonging to the TENA/THI-4 family of proteins, could play a role in compensating the fitness cost of erythromycin resistance through a positive relationship with flagellar proteins (Hao et al.). The protein C 1199 seems to be involved in the leucine biosynthesis and transport but could also indirectly affect the development of erythromycin resistance in $C$. jejuni (Hao et al.). In another study also published in this research topic (Taylor et al.), the analysis of two putative chaperone genes (Cj1289 and Cj0694) was investigated using a mutational approach. The protein Cj0694 is predicted as an inner membrane anchored protein but possesses a peptidylprolyl cis/trans isomerase (PPIase) activity, which could be involved in the initial folding and outer membrane translocation of Cj1289, a SurA-like chaperone (SalC). These two proteins likely participate to the outer membrane protein biogenesis and integrity.

\section{Methodology to Improve Cultural Technique}

Culture-based isolation methods targeting Campylobacter spp. are usually challenging for some type of highly contaminated samples, due to its outgrowth by major competing bacteria in the enrichment conditions. In this research topic, an improved culture-dependent methodology for the selective isolation of $C$. jejuni from wastewater samples is described. Kim et al. assessed a few different enrichment conditions using five different antibiotics (i.e., cefoperazone, vancomycin, trimethoprim, polymyxin B, and rifampicin), to which C. jejuni is intrinsically resistant. They showed that Enterococcus spp. and Pseudomonas aeruginosa are major competing bacteria in the 
enrichment conditions and that the addition of polymyxin $\mathrm{B}$, rifampicin or both to the selective media enhanced the selective isolation of C. jejuni.

\section{Chicken Gut Microbiota Challenged with Campylobacter}

The gut microbiota plays an essential role in nutrition, feed conversion, growth performance and protection against pathogenic bacteria such as Campylobacter spp. However, and despite the increasing number of articles focused on the gut microbiome of humans and animals, there is little information yet about the diversity and function of the gut microbiota in chickens and its impact on the establishment of certain pathogens, including Campylobacter spp. This research topic includes a couple of articles dealing with this issue. Thibodeau et al. assessed the impact of feed supplementation with selenium on the gut microbiota of chickens in a C. jejuni colonization model. Results obtained by these authors evidenced that, for healthy chickens raised in good hygienic conditions, seleniumyeast did not influence neither the body weight nor the caecal microbiota or the colonization status by $C$. jejuni. Awad et al. monitored in a longitudinal study from day 1 to day 28 of age the composition and structure of the

\section{REFERENCES}

Boysen, L., Wechter, N. S., and Rosenquist, H. (2013). Effects of decontamination at varying contamination levels of Campylobacter jejuni on broiler meat. Poult. Sci. 92, 1425-1429. doi: 10.3382/ps.2012-02889

Connerton, P. L., Timms, A. R., and Connerton, I. F. (2011). Campylobacter bacteriophages and bacteriophage therapy. J. Appl. Microbiol. 111, 255-265. doi: 10.1111/j.1365-2672.2011.05012.x

EFSA and ECDC (2016). European Food Safety Authority, European Centre for Disease Prevention and Control: The European Union summary report on trends and sources of zoonoses, zoonotic agents and food-borne outbreaks in 2014. EFSA J. 13:4329. doi: 10.2903/j.efsa.2015.4329

Ghaffar, N. M., Connerton, P. L., and Connerton, I. F. (2015). Filamentation of Campylobacter in broth cultures. Front. Microbiol. 6:657. doi: 10.3389/fmicb.2015.00657

Hue, O., Le Bouquin, S., Laisney, M.-J., Allain, V., Lalande, F., Petetin, I., et al. (2010). Prevalence of and risk factors for Campylobacter spp. contamination of broiler chicken carcasses at the slaughterhouse. Food Microbiol. 27, 992-999. doi: 10.1016/j.fm.2010.06.004

Lawes, J. R., Vidal, A., Clifton-Hadley, F. A., Sayers, R., Rodgers, J., Snow, L., et al. (2012). Investigation of prevalence and risk factors for Campylobacter in broiler flocks at slaughter: results from a UK survey. Epidemiol. Infect. 140, 1725-1737. doi: 10.1017/S0950268812000982

Macé, S., Haddad, N., Zagorec, M., and Tresse, O. (2015). Influence of measurement and control of microaerobic gaseous atmospheres in methods for Campylobacter growth studies. Food Microbiol. 52, 169-176. doi: 10.1016/j.fm.2015.07.014

McFadyean, J., and Stockman, S. (1913). Report of the Departmental Committee Appointed by the Board of Agriculture and Fisheries to Inquire into Epizootic Abortion. Part III. Abortion in Sheep. HMSO London.

Powell, L. F., Lawes, J. R., Clifton-Hadley, F. A., Rodgers, J., Harris, K., Evans, S. J., et al. (2012). The prevalence of Campylobacter spp. in broiler flocks and on broiler carcases, and the risks associated with highly contaminated carcases. Epidemiol. Infect. 140, 2233-2246. doi: 10.1017/S09502688120 00040

Rodrigues, R. C., Haddad, N., Chevret, D., Cappelier, J.-M., and Tresse, O. (2016). Comparison of proteomics profiles of Campylobacter jejuni strain microbiota of the gut content and the mucosa, as well as the consequences of a $C$. jejuni infection on the gut microbiome. These authors show in their article that the chicken gut microbiota significantly changes during the first 28 days of age, that numerous significant differences in microbial profiles are observed between the mucosa and luminal content of the small and large intestine, and that $C$. jejuni colonization is associated with an alteration of the gut microbiota, which confirms that the Campylobacter carrier state in chickens is characterized by multiple changes in the intestinal ecology within the host.

\section{CONCLUSION}

Recent researches indicate that genomic polymorphism, restricted catabolic capacity, self-regulation or deregulation of genes, bacterial cooperation and unknown contamination routes may all be connected to the specificity of pathogenic species of Campylobacter.

\section{AUTHOR CONTRIBUTIONS}

OT prepared the content. OT, IC, and AA wrote the paper.
Bf under microaerobic and aerobic conditions. Front. Microbiol. 7:1596. doi: 10.3389/fmicb.2016.01596

Rodrigues, R. C., Pocheron, A. L., Hernould, M., Haddad, N., Tresse, O., and Cappelier, J. M. (2015). Description of Campylobacter jejuni Bf, an atypical aero-tolerant strain. Gut Pathog. 7, 30. doi: 10.1186/s13099-015-0077-x

Sellars, M. J., Hall, S. J., and Kelly, D. J. (2002). Growth of Campylobacter jejuni supported by respiration of fumarate, nitrate, nitrite, trimethylamineN-oxide, or dimethyl sulfoxide requires oxygen. J. Bacteriol. 184, 4187-4196. doi: 10.1128/jb.184.15.4187-4196.2002

Stahl, M., Friis, L. M., Nothaft, H., Liu, X., Li, J., Szymanski, C. M., et al. (2011). Lfucose utilization provides Campylobacter jejuni with a competitive advantage. Proc. Natl. Acad. Sci. U.S.A. 108, 7194-7199. doi: 10.1073/pnas.1014125108

Tangwatcharin, P., Chanthachum, S., Khopaibool, P., and Griffiths, M. W. (2006). Morphological and physiological responses of Campylobacter jejuni to stress. J. Food Prot. 69, 2747-2753.

Vandamme, P., Falsen, E., Rossau, R., Hoste, B., Segers, P., Tytgat, R., et al. (1991). Revision of Campylobacter, Helicobacter, and Wolinella taxonomy: emendation of generic descriptions and proposal of Arcobacter gen. nov. Int. J. Syst. Bacteriol. 41, 88-103. doi: 10.1099/00207713-41-1-88

Véron, M., and Chatelain, R. (1973). Taxonomic study of the genus Campylobacter Sebald and Véron and designation of the neotype strain for the type species, Campylobacter fetus (Smith and Taylor) Sebald and Véron. J. System. Bacteriol. 23, 122-134. doi: 10.1099/00207713-23-2-122

Weingarten, R. A., Taveirne, M. E., and Olson, J. W. (2009). The dual-functioning fumarate reductase is the sole succinate: quinone reductase in Campylobacter jejuni and is required for full host colonization. J. Bacteriol. 191, 5293-5300. doi: 10.1128/JB.00166-09

Conflict of Interest Statement: The authors declare that the research was conducted in the absence of any commercial or financial relationships that could be construed as a potential conflict of interest.

Copyright (C) 2017 Tresse, Alvarez-Ordóñez and Connerton. This is an open-access article distributed under the terms of the Creative Commons Attribution License (CC $B Y)$. The use, distribution or reproduction in other forums is permitted, provided the original author(s) or licensor are credited and that the original publication in this journal is cited, in accordance with accepted academic practice. No use, distribution or reproduction is permitted which does not comply with these terms. 